We would also like to thank Singh and Stewart for their interest and response to our article. Although it is true that the relative risk of anaphylaxis to nondepolarizing muscle relaxants is high compared to other anaesthetic agents, it is a small risk in absolute terms (estimated to be 1:15000-30000 anaesthetics). We would argue this is considerably lower than the risk of sight-threatening intraoperative complication due to the eccentric eye.

They are also quite correct in that local anaesthesia infiltrations may relieve the problem. They also may not. Furthermore, peribulbar injections carry the risk of globe perforation.

We would maintain that our proposed solution is a reliable, reasonable and safe one, particularly when a 'tincture of time' is either unavailable or ineffective.

JD Rossiter and M Wood

Department of Ophthalmology, Taunton and Somerset NHS Trust, Taunton, Somerset, UK

Correspondence: JD Rossiter,

Tel: +44 1823 342950;

Fax: +44 1823342943

E-mail: jonathan.rossiter@tst.nhs.uk

Eye (2007) 21, 257-258. doi:10.1038/sj.eye.6702490; published online 21 July 2006

Sir,

\section{Blebitis after deep sclerectomy}

Blebitis after trabeculectomy is a well known and potentially dangerous infection that can lead to endophthalmitis as the normal barriers for intraocular spread of bacteria are weakened. ${ }^{1}$

In deep sclerectomy, there is no penetrating opening intraocularly as Descemet's membrane and the trabecular meshwork are still intact at the site of filtration. To the best of our knowledge, blebitis after deep sclerectomy has not been described before. Here, we report a case of blebitis with severe intraocular inflammation after deep sclerectomy.

\section{Case report}

A 59-year-old Caucasian male presented with a pseudoexfoliative glaucoma in his right eye (RE). His intraocular pressure (IOP) was $56 \mathrm{mmHg}$ and he had already sustained severe damage to the visual field and the optic disc. He was referred to our clinic for glaucoma surgery when medical treatment was insufficient to control IOP. At the first visit at our clinic, his RE had an IOP of $39 \mathrm{mmHg}$ despite medical treatment and visual acuity (VA) was 6/12.

A standard deep sclerectomy was performed. Preoperatively, the eye was rinsed with chlorhexidine solution $0.05 \% .^{2}$ After sub-Tenon's anaesthesia, a limbal peritomy was performed and the conjunctiva was retracted. An outer scleral flap was created and a deep internal scleral triangular flap was dissected anteriorly until the canal of Schlemm was opened and HealonGV was injected into the canal on each side. The internal wall of Schlemm's canal was peeled off. Anteriorly, the Descemet's membrane was dissected from the corneal stroma for about $1 \mathrm{~mm}$ and good percolation of aqueous humour was observed. The internal scleral flap was excised and an implant was sutured to the bottom of the scleral 'lake'. The implant was a $4 \mathrm{~mm}$ cut piece of a 1-0 polydioxanone ligature (Ethicon PDS*II article number Z627E). The outer flap was sutured with two single nylon sutures and the conjunctiva was closed with a vicryl suture. Two milligrams of betamethasone were injected subconjunctivally. No antibiotics were used either pre-, intra-, or postoperatively, which is standard in sclerectomy surgery in our institution. No antimetabolite was used intra- or postoperatively.

Postoperatively, the eye was treated with dexamethasone $0.1 \%$ q.i.d. starting directly after the operation.

The first postoperative days were uneventful with IOP $2 \mathrm{mmHg}$ on day 1 and $12 \mathrm{mmHg}$ on day 3 .

On postoperative day 13, the patient reported pain and redness and eyelid swelling with some purulent secretion from the eye with an onset on the previous day. On slit-lamp examination, the eye was red with swollen eyelids, and purulent secretion was noted around the bleb. The IOP was $11 \mathrm{mmHg}$, VA was $6 / 24$ and there were $3+$ cells in the anterior chamber. A conjunctival smear culture was taken and the patient was treated with topical levofloxacin $0.05 \%$ drops every hour.

Sixteen hours later, he was re-examined. The eye showed increased injection with a whitish filtration bleb surrounded by dilated hyperemic vessels and purulent secretion (Figure 1). There were $4+$ cells in the anterior chamber with fibrinous strands but no hypopyon. The vitreous was clear, however. The IOP was $20 \mathrm{mmHg}$. He was admitted to hospital and treated topically with fortified cefuroxime $3 \%$ and levofloxacin $0.05 \%$ every hour, cyclopentolate $1 \%$ t.i.d. and he continued dexamethasone $0.1 \%$ q.i.d. Intraocular samples were not taken as overt endophthalmitis was not suspected. ${ }^{1}$ The conjunctival culture was positive for Staphylococcus 


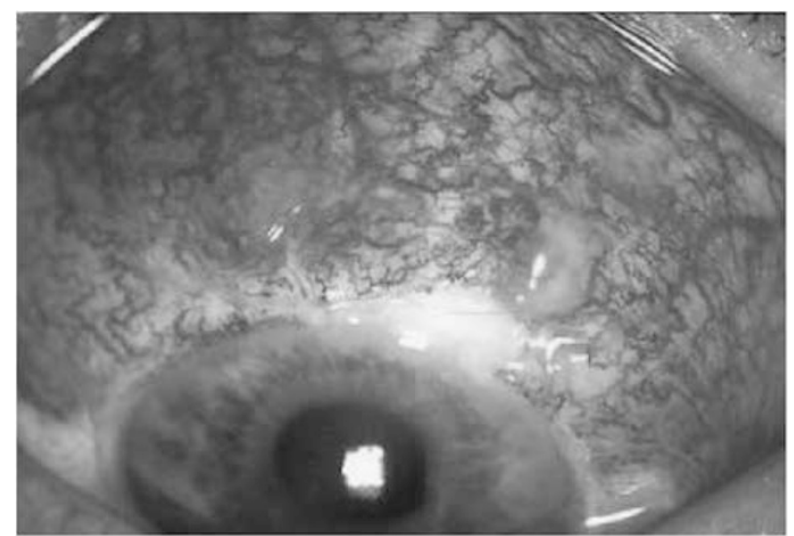

Figure 1 The eye on day 14. Purulent secretion is seen around the conjunctival sutures and inside the greyish limbal bleb with intense injection of the conjunctiva.

aureus, sensitive for levofloxacin, isoxazolylpenicillin, gentamicin, chloramfenicol, fusidic acid, and tetracycline. The inflammation abated in the following days, an IOP rise to $36 \mathrm{mmHg}$ was successfully treated with a combination drop of timolol $0.5 \%+$

dorzolamide $2 \%$.

The patient was released from the hospital after 6 days. One month after surgery, VA was $6 / 20$, the IOP was $10 \mathrm{mmHg}$, and treatment was furthered tapered during the following 2 months.

Four months postoperatively, the IOP was $24 \mathrm{~mm}$ with some filtration. A gonioscopy showed no visible microperforation of the internal trabeculo-Descemet's membrane. A successful YAG goniopuncture was performed on that occasion.

Six months after surgery, VA was $6 / 12$ and the IOP was $15 \mathrm{mmHg}$ without medical therapy and with a nice broad functioning filtration bleb with no anterior chamber reaction and a clear vitreous.

\section{Comments}

Deep sclerectomy and other nonperforating glaucoma surgeries are rising in numbers all over the world as this approach compared to the standard trabeculectomy has a lower incidence of severe hypotony in the immediate postoperative period. ${ }^{3}$ In deep sclerectomy, as opposed to viscocanalostomy, a filtration bleb is desired to keep the IOP regulated.

To the best of our knowledge, blebitis after nonperforating filtration surgery has not been reported before and we therefore find it interesting to report this case. Our patient had no visible microperforation of the trabeculo-Descemet's membrane during the operation. We must admit, however, that it cannot be ruled out that microperforation did indeed occur, for example, with the
HealonGV cannula in the internal wall of the canal of Schlemm outside the visible parts of the operation area. Even though the gonioscopy at 4 months postoperatively showed no signs of microperforation, it is possible that it could have healed before.

Just like in infectious keratitis or infectious blebitis following trabeculectomy, there should be a risk for intraocular infectious spread from a blebitis associated with deep sclerectomy. ${ }^{1,4}$ Although differentiating between a blebitis and an overt endophthalmitis remains a clinical challenge, conditions with pus in the filtration bleb, moderate anterior chamber inflammation, a modest decrease in VA, and no evident vitreous inflammation are regarded as blebitis signs in our clinic. In such cases, a conjunctival smear for culture and topical antibiotics are judged sufficient, ${ }^{1,5}$ but one group has reported a high yield of vitreous positive cultures in the absence of evident vitritis. ${ }^{6}$ In the present case, it was decided to observe the patient in the hospital while no intraocular interventions were considered necessary as the patient improved.

The implant used herein ${ }^{7}$ is not commercially available for this indication, but is used for sclerectomy in some Swedish clinics. Preliminary experience of the device has been presented as posters at various congresses. The polydioxanone material has not been associated with a higher incidence of infection in other studies. ${ }^{8,9}$

Finally, our prophylactic protocol needs commenting. As there are no controlled studies supporting the prophylactic efficacy of antibiotics of any kind in glaucoma surgery, our antimicrobial treatment for deep sclerectomy has been limited to chlorhexidine solution rinsing of the conjunctiva. Only time will tell whether sclerectomy patients with or without an implant is at the same risk as trabeculectomized patients to develop blebitis or even endophthalmitis.

\section{Acknowledgements}

Proprietary interests: None.

\section{References}

1 Ciulla TA, Beck AD, Topping TM, Sullivan Baker A. Blebitis, early endophthalmitis, and late endophthalmitis after glaucoma-filtering surgery. Ophthalmology 1997; 104: 986-995.

2 Montan PG, Setterquist H, Marcusson E, Rylander M, Ransjö U. Preoperative gentamicin eye drops and chlorhexidine solution in cataract surgery. Experimental and clinical results. Eur J Ophthalmol 2000; 10: 286-292.

3 El Sayyad F, Helal M, El-Kholify H, Khalil M, El-Maghraby A. Nonpenetrating deep sclerectomy versus trabeculectomy in bilateral primary open-angle glaucoma. Ophthalmology 2000; 107: 1671-1674. 
4 Scott I, Flynn H, Feuer W, Pflugfelder S, Alfonso E, Forster R et al. Endophthalmitis associated with microbial keratitis. Ophthalmology 1996; 103: 1864-1870.

5 Chen PP, Gedde SJ, Budenz DL, Parrish RK. Outpatient treatment of bleb infection. Arch Ophthalmol 1997; 115: 1124-1128.

6 Waheed S, Ritterband DC, Greenfield DS, Liebmann JM, Seedor JA, Ritch R. New patterns of infecting organisms in late bleb-related endophthalmitis: a ten year review. Eye 1998; 12: 910-915.

7 Wallin O. Comparison of different implants for deep sclerectomy. Acta Ophthalmologica Scandinavia 2004; 82: 345 (XXXVI Nordic Congress of Ophthalmology, Abstracts. Poster available by e-mail to orjan.wallin@sankterik.se).

8 Krukowski ZH, Cusick EL, Engeset J, Matheson NA. Polydioxanone or polypropylene for closure of midline abdominal incisions: a prospective comparative clinical trial Br J Surg 1987; 74: 828-830.

9 Paterson-Brown S, Cheslyn-Curtis S, Biglin J, Dye J, Easmon CS, Dudley HA et al. Suture materials in contaminated wounds: a detailed comparison of a new suture with those currently in use. Br J Surg 1987; 74: 734-735.

\section{OJI Wallin and PG Montan}

Department of Anterior Segment Surgery, St Erik Eye Hospital, Polhemsgatan 50, SE-11282,

Stockholm, Sweden

Correspondence: O Wallin

St Erik Eye Hospital,

Polhemsgatan 50, SE-112 82 Stockholm, Sweden

Tel: + 468672 3000;

Fax: + 4686510785 .

E-mail: orjan.wallin@sankterik.se

Eye (2007) 21, 258-260. doi:10.1038/sj.eye.6702494;

published online 23 June 2006

Sir,

Regarding Newton's laws (Editorial, Eye, May 2006)

I read the Editorial about pathways for macular disease with interest. ${ }^{1}$ Can I correct one point?

The misquoted law should have read Newton's Third Law of Motion. No thermodynamic laws are attributed to him. For completeness, Newton's Laws of Motion and the Laws of Thermodynamics are as follows:

Newton's First Law: An object at rest tends to stay at rest and an object in uniform motion tends to stay in uniform motion unless acted upon by a net external force.

Newton's Second Law: An applied force equals the rate of change of momentum.

Newton's Third Law: For every action there is an equal and opposite reaction.
Zeroth Law of Thermodynamics: If $\mathrm{A}$ and $\mathrm{B}$ are in thermal equilibrium, and $\mathrm{B}$ and $\mathrm{C}$ are also in thermal equilibrium, then $\mathrm{A}$ and $\mathrm{C}$ are in thermal equilibrium.

First Law of Thermodynamics: The increase in the energy of a closed system is equal to the amount of energy added to the system by heating, minus the amount lost in the form of work done by the system on its surroundings.

Second Law of Thermodynamics: The total entropy of any isolated thermodynamic system tends to increase over time, approaching a maximum value.

Third Law of Thermodynamics: As a system approaches absolute zero of temperature, all processes cease and the entropy of the system approaches a minimum value or zero for the case of a perfect crystalline substance.

\section{Reference}

1 Ellis J, Cole A, Roxburgh STD. Patient pathways for macular disease: what will the new optometrist with special interest achieve? Eye 2006; 20: 521-522.

N Davies

Department of Ophthalmology, Chelsea \& Westminster Hospital, 369 Fulham Road, London SW10 9NH, UK

Correspondence: N Davies,

Tel: + 4402087 468345;

Fax: + 4402082375040

E-mail: nigel@npdavies.wanadoo.co.uk

Eye (2007) 21, 260. doi:10.1038/sj.eye.6702497;

published online 23 June 2006

Sir,

Xanthogranuloma of the lacrimal sac as a manifestation of Wegener's granulomatosis

Wegener's granulomatosis (WG) is a multisystemic disease that affects small vessels. The necrotizing granulomatous inflammation commonly involves the upper airways, lung, and kidney, although any organ can be affected. ${ }^{1}$ Xanthogranuloma formation is a rare manifestation of the disease. ${ }^{1}$

Ocular manifestations occurs in approximately $30-50 \%$ of cases. ${ }^{2}$ The lacrimal drainage system is usually affected by direct spread of the inflammatory process from the upper airways. ${ }^{3}$ Focal vasculitis of the lacrimal 